PAPER

\section{Orbit analysis of a geostationary gravitational wave interferometer detector array}

To cite this article: Massimo Tinto et al 2015 Class. Quantum Grav. 32185017

View the article online for updates and enhancements.

\section{Related content}

- Milli-Hertz Gravitational Waves: LISA and
$\frac{\text { LISA PathFinder }}{\text { H Araújo, P Cañizares, M Chmeissani et }}$
al.
- TianQin: a space-borne gravitational wave
$\frac{\text { detector }}{\text { Jun Luo, Li-Sheng Chen, Hui-Zong Duan }}$
et al.
- Topical Review
O Jennrich

\section{Recent citations}

- Pierre Bourget et al

- Enhanced Gravitational Wave Science with LISA and gLISA. Massimo Tinto

- Coherent observations of aravitational radiation with LISA and gLISA Massimo Tinto et al

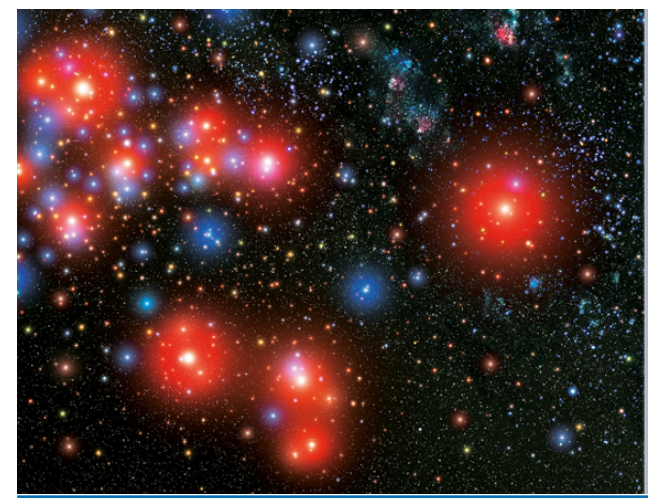

\title{
$\frac{\mathbf{A}}{\mathbf{S}}$ IOP Astronomy ebooks
}

Part of your publishing universe and your first choice for astronomy, astrophysics, solar physics and planetary science ebooks. iopscience.org/books/aas 


\title{
Orbit analysis of a geostationary gravitational wave interferometer detector array
}

\author{
Massimo Tinto ${ }^{1,4}$, Jose C N de Araujo ${ }^{2}$, Helio K Kuga ${ }^{2}$, \\ Márcio E S Alves ${ }^{3}$ and Odylio D Aguiar ${ }^{2}$ \\ ${ }^{1}$ Jet Propulsion Laboratory, California Institute of Technology, Pasadena, 91109, \\ CA, USA \\ ${ }^{2}$ Instituto Nacional de Pesquisas Espaciais, S. J. Campos, SP, Brazil \\ ${ }^{3}$ Instituto de Ciência e Tecnologia, UNESP-Univ. Estadual Paulista, S. J. Campos, \\ SP, Brazil \\ E-mail: massimo.tinto@jpl.nasa.gov, jcarlos.dearaujo@inpe.br, hkk@dem.inpe.br, \\ marcio.alves@ict.unesp.br and odylio.aguiar@inpe.br
}

Received 20 October 2014, revised 7 June 2015

Accepted for publication 27 July 2015

Published 2 September 2015

\begin{abstract}
We analyze the trajectories of three geostationary satellites forming the geostationary gravitational wave interferometer (GEOGRAWI) [1], a spacebased laser interferometer mission aiming to detect and study gravitational radiation in the $\left(10^{-4}-10\right) \mathrm{Hz}$ band. The combined effects of the gravity fields of the Earth, the Sun and the Moon make the three satellites deviate from their nominally stationary, equatorial and equilateral configuration. Since changes in the satellites's relative distances and orientations could negatively affect the precision of the laser heterodyne measurements, we have derived the timedependence of the inter-satellite distances and velocities, the variations of the polar angles made by the constellation's three arms with respect to a chosen reference frame and the time changes of the triangle's enclosed angles. We find that during the time between two consecutive station-keeping maneuvers (about two weeks) the relative variations of the inter-satellite distances do not exceed a value of $0.05 \%$, while the relative velocities between pairs of satellites remain smaller than about $0.7 \mathrm{~m} \mathrm{~s}^{-1}$. In addition, we find the angles made by the arms of the triangle with the equatorial plane to be periodic functions of time whose amplitudes grow linearly with time; the maximum variations experienced by these angles as well as by those within the triangle remain smaller than 3 arc-minutes, while the east-west angular variations of
\end{abstract}

4 Author to whom any correspondence should be addressed. 
the three arms remain smaller than about 15 arc-minutes during the two-week period.

Keywords: gravitational waves, interferometry, geostationary satellites

\section{Introduction}

Gravitational waves (GWs), predicted by Einstein's theory of general relativity, are disturbances of space-time propagating at the speed of light. Because of their extremely small cross-sections, GWs carry information about regions of the Universe that would be otherwise unobtainable through the electromagnetic spectrum. Once detected, GWs will allow us to open a new observational window on the Universe, and perform a unique test of general relativity [2].

Since the first pioneering experiments by Joseph Weber in the early sixties [3], several experimental groups around the world have been attempting to detect GWs. The coming online of the Advanced LIGO and Advanced Virgo detectors [4, 5], however, is likely to break this sequence of experimental drawbacks and result into the first detection before the end of this decade.

Contrary to ground-based detectors, which are sensitive to GWs in a band from about a few tens of $\mathrm{Hz}$ to a few kilohertz, space-based interferometers are expected to access a much lower frequency region (from a few tenths of millihertz to about a few tens of $\mathrm{Hz}$ ) where $\mathrm{GW}$ signals are expected to be larger in number and characterized by larger amplitudes. The most notable example of a space interferometer, which for several decades has been jointly studied in the United States of America and in Europe, is the Laser Interferometer Space Antenna (LISA) mission [6]. By relying on coherent laser beams exchanged between three remote spacecraft forming a giant (almost) equilateral triangle of arm-length equal to $5 \times 10^{6} \mathrm{~km}$, LISA aimed to detect and study cosmic GWs in the $10^{-4}-1 \mathrm{~Hz}$ band.

Although over the years only a few space-based detector designs have been considered as alternatives to the LISA mission [7-9] starting in 2011 other mission concepts have appeared in the literature [10] in conjunction with the ending of the NASA/ESA partnership for flying LISA. Their goals were to meet (at a lower cost) most (if not all) the LISA scientific objectives (highlighted in the 2010 Astrophysics Decadal Survey New Worlds, New Horizons [11]).

Geostationary gravitational wave interferometer (GEOGRAWI) was one of the alternative concepts to the LISA mission submitted in response to NASA's request for information \# NNH11ZDA019L [12]. It entails three spacecraft in geostationary orbit, forming an equilateral triangle of arm-length approximately equal to $73000 \mathrm{~km}$. Like LISA, it has three identical spacecraft exchanging coherent laser beams but, by being in a geostationary orbit, it achieves its best sensitivity in a frequency band $\left(3 \times 10^{-2}-1 \mathrm{~Hz}\right)$ [1] that is complementary to those of LISA and ground detectors. The astrophysical sources that GEOGRAWI is expected to observe within its operational frequency band include extra-galactic massive and supermassive black-hole coalescing binaries, the resolved galactic binaries and extra-galactic coalescing binary systems containing white dwarfs and neutron stars, a stochastic background of astrophysical and cosmological origin, and possibly more exotic sources such as cosmic strings. GEOGRAWI will be able to test Einstein's theory of relativity by comparing the waveforms detected against those predicted by alternative relativistic theories of gravity, and also by measuring the number of independent polarizations of the detected GW signals [13]. 
It has recently been proposed [14] to fly a GEOGRAWI mission by taking advantage of the NASA science instrument hosting program [15] onboard commercial geostationary satellites (comsats). Several aerospace companies, such as Space Systems Loral, Boeing, Lockheed, and others, offer to fly for a fee (which depends on the instrument's mass, power, and complexity) additional payload in the form of scientific instruments on their planned comsats. They are deployed at a rate of about 20 per year worldwide. Comsat fleet operators will launch to positions around the globe to achieve telecom world coverage. Therefore, opportunities exist to host GW payloads on three comsats in three longitudes roughly equally spaced. These comsats have large resources of power and cargo space, especially early in mission life and, as the scientific mission life is less than the $15 \mathrm{yr}$ comsat life, it allows the hosted payload to utilize early life system margin. Comsats can weigh more than 5 ton, easily carry $300 \mathrm{~kg}$ of additional mass, and transmit the science data to their ground stations at a very high rate. By accessing these platforms we will save the costs associated with the satellites, the launching vehicle, the onboard and ground telecom systems, and minimize part of the engineering costs inherent with the construction and integration of the entire mission. One of the drawbacks of flying onboard comsats rather than relying on dedicated satellites is that they perform regular operations of 'station-keeping' [16] to compensate for the gravitational perturbations exerted by the Sun, the Moon and the gravity field of the Earth. These trajectory adjustments, which take place about every two weeks and last for about an hour, allow them to maintain their locations relative to the ground fixed. During these orbit adjustment periods the onboard drag-free subsystem will need to be re-caged to prevent possible damages to occur ${ }^{5}$. It should be said, however, that the station-keeping maneuvers performed by the three satellites are particularly important as they offset the excessive variations of the inter-spacecraft relative orientations and velocities and maintain the constellation in a stable configuration. If the spacecraft would be left to drift apart for periods longer than the station-keeping duty cycle, the quality of the laser heterodyne measurements performed by the constellation would start to degrade, resulting into a degradation of the science objectives of GEOGRAWI [1].

Our paper analyzes the time evolution of the GEOGRAWI constellation during the time between two station-keeping maneuvers, and it is organized as follows. In section 2 we derive the trajectory of each spacecraft (as a function of time) by numerically solving the Newtonian equations of motion of a 'point-particle' in the gravitational potentials of the Earth, the Moon and the Sun. After noticing that the effects of the solar radiation pressure on each spacecraft are not included into the equations of motion because they are compensated for by the spacecraft drag-free system, in section 2.1 we estimate the magnitude of the variations of the inter-spacecraft distances, velocities, and relative angular orientations. We find that, during the time between two consecutive station-keeping maneuvers (about two weeks), the relative variations of the inter-satellite distances do not exceed a value of $0.05 \%$, while the relative velocities between pairs of satellites remain smaller than about $0.7 \mathrm{~m} \mathrm{~s}^{-1}$. In addition, we find the angles made by the arms of the triangle with the equatorial plane to be periodic functions of time whose amplitudes grow linearly with time; the maximum variations experienced by these angles as well as by those within the triangle remain smaller than 3 arc-minutes, while the east-west angular variations of the three arms remain smaller than about 15 arc-minutes during a two-week period.

5 To take advantage of these large and heavy satellites for the purpose of detecting and studying gravitational radiation in the $\mathrm{mHz}$ band a new drag-free system has been identified [14], which should allow GEOGRAWI to achieve the desired degree of inertia. 


\section{Orbit dynamics}

GEOGRAWI measures relative frequency changes experienced by coherent laser beams exchanged by its three pairs of spacecraft. As the laser beams are received, they are made to interfere with the outgoing laser light. These heterodyne measurements are each downconverted with the use of an onboard oscillator, then digitized and numerically combined in order to cancel the lasers frequency fluctuations [17]. The spacecraft are made to follow an orbit determined by the gravitational forces on a (spherical) test mass (located onboard each spacecraft) due to the Earth, the Moon and the Sun and are therefore drag-free. Since the relative distances between spacecraft are not constant, the heterodyne measurements will display a resulting Doppler shift, which is then removed from the data by using an onboard clock. The magnitude of the frequency fluctuations introduced by the clocks into the heterodyne measurements depends linearly on the noises from the clocks themselves and the inter-spacecraft relative velocities. Space-qualified, state of the art clocks are oven-stabilized crystals characterized by an Allan deviation of $\sigma_{\mathrm{A}} \approx 10^{-13}$ for averaging times of $1-1000 \mathrm{~s}$, covering most of the frequency band of interest to GEOGRAWI. The corresponding power spectral density of the relative frequency fluctuations, $S_{y}(f)$, associated with this 'flicker-noise' at the Fourier frequency $f$ is given by the following expression [18]

$$
S_{y}(f)=\frac{\sigma_{\mathrm{A}}^{2}}{2 \ln 2} \frac{\nu_{\mathrm{D}}^{2}}{\nu_{0}^{2}} f^{-1} \mathrm{~Hz}^{-1},
$$

where we have denoted with $\nu_{\mathrm{D}}$ the frequency change induced by the Doppler effect on the one-way heterodyne measurement and with $\nu_{0}$ the nominal laser frequency. Since $\nu_{\mathrm{D}}$ is equal to $\nu_{\mathrm{D}}=\nu_{0} v / c$, with $v$ being the two spacecraft relative velocity and $c$ the speed of light, we can rewrite equation (1) in the following form

$$
S_{y}(f)=\frac{\sigma_{\mathrm{A}}^{2}}{2 \ln 2} \frac{v^{2}}{c^{2}} f^{-1} \mathrm{~Hz}^{-1} \text {. }
$$

If we take a spacecraft relative velocity of $0.7 \mathrm{~m} \mathrm{~s}^{-1}$ (which we derive in the following subsection 2.1), a Fourier frequency $f=10^{-3} \mathrm{~Hz}$, and the frequency of the laser to be equal to $\nu_{0}=3.0 \times 10^{14} \mathrm{~Hz}$, we find a $S_{y}\left(10^{-3}\right)=3.9 \times 10^{-41} \mathrm{~Hz}^{-1}$. To put this number in perspective, in reference [1] we showed that at this frequency the GEOGRAWI noise level goal (determined by our estimate of the remaining noises) was equal to about $3.7 \times 10^{-46}$, roughly five orders of magnitude smaller than the above estimated noise due to the clock. Recent developments in the design of space-qualified clocks [19] indicate that oscillators capable of an Allan deviation of a few parts in $10^{-15}$ or better at $1000 \mathrm{~s}$ integration time will be available by the end of this decade. Such a clock performance would still require the implementation of the clock-noise calibration procedure [20] with an inter-spacecraft velocity of $0.7 \mathrm{~m} \mathrm{~s}^{-1}$ since the resulting clock noise spectrum would still be about a factor of 10 or so above the GEOGRAWI noises. It should be said, however, that the resulting set of performance requirements levied on the onboard subsystems involved in the clock calibration procedure will be relatively easy to meet $[6,21,22]$.

Besides the magnitude of the inter-spacecraft relative velocities, the spacecraft ability of properly pointing to each other is obviously important. In order to address the above questions in the contest of GEOGRAWI, in what follows we first integrate the equations of motion for each of the three GEOGRAWI spacecraft. At an arbitrarily chosen starting time $t=0$, we assume the spacecraft to be at rest with respect to a right-handed, orthogonal coordinate system associated with a reference frame that rotates jointly with the Earth. In it the Z-axis coincides with the Earth's axis of rotation, the $X$-axis intersects the Greenwich line in the 
equatorial plane, and the $Y$ axis is orthogonal to it. Under the influence of the Earth, Moon, and Sun gravitational fields, the three spacecraft move from their starting locations corresponding to an equilateral triangle configuration.

Since the Earth is much closer to the spacecraft than the Moon and the Sun, its gravitational potential has to be described mathematically in such a way to reflect the Earth's mass non-spherical and non-symmetrical distribution. This uneven distribution of mass is expressed by the so-called coefficients of spherical harmonics, which enter into the multi-pole expansion of the Earth's gravitational potential (discussed in the appendix). In order to have high accuracy, and because it was also easy to do, our model of the Earth's gravitational potential relied on the EGM2008, which was made to include terms in the multi-pole expansion up to order/degree 2100 [23]. By relying on our numerical integrator [24], we derived the trajectories of the three satellites, i.e. their location and velocity vectors as functions of time. In what follows we provide a description of the kinematic quantities of relevance to GEOGRAWI that we have been able to derive, i.e. the time-dependence of the inter-satellite distances and velocities, and their relative angular orientations.

\subsection{Inter-satellite distances, velocities, and relative orientations}

Before describing our estimated relative changes of the three arm-lengths, we should first remind ourselves that the gravity field of the Earth is not invariant under rotation around the Earth's rotation axis because of its non-spherical and non-symmetrical mass distribution. This implies that the variation of the inter-spacecraft distances will depend on the initial longitudinal configuration of the constellation, opening the possibility for the existence of a specific starting longitude of the equilateral triangle minimizing the maximum of the interspacecraft velocities. Although there exist a specific value of the longitude of the constellation resulting into a mini-max value of the relative velocities, the resulting reduction in the relative velocities is not significant enough to make it a mission requirement.

In figure 1 we plot the time-dependence of the three inter-spacecraft distances relative to the nominal arm-length of the equilateral triangle at time $t=0\left(7.3 \times 10^{4} \mathrm{~km}\right)$. The plot covers a period of thirty days during which no station-keeping maneuvers have been accounted for. Since these typically happen about once or twice per fortnight, we conclude that during the first, say, 15 days the maximum relative variations of the three inter-spacecraft distances do not exceed a value of $0.05 \%$. Although such small variations of the relative distances, together with the resulting arm-length inequalities, still require GEOGRAWI to rely on time-delay interferometry [17] for canceling the laser frequency fluctuation, more stable coherent lasers expected to become space-qualified before the end of this decade [25] should allow us to operate GEOGRAWI like a ground-based interferometer.

In figure 2 we now plot the time-dependence of the inter-spacecraft relative velocities, again for a period of 30 days. Note these three time-series are periodic functions of time with period equal to one day and amplitudes that depend on time and do not exceed a maximum value of about $0.7 \mathrm{~m} \mathrm{~s}^{-1}$. This is still large enough to require the use of the calibration procedure for canceling the noise from current space-qualified clocks in the GEOGRAWI interferometric measurements [20].

In figure 3 we now plot the variation of the angles enclosed by the triangular constellation. The values shown correspond to the differences between each angle's value at time $t$ and the $60^{\circ}$ value at time $t=0$. During the first two weeks the enclosed angles do not change much, remaining within the \pm 3 arc-minute range. To put this number in perspective, the diffraction limit of a $10 \mathrm{~cm}$ telescope GEOGRAWI might use corresponds to about 4 arc- 


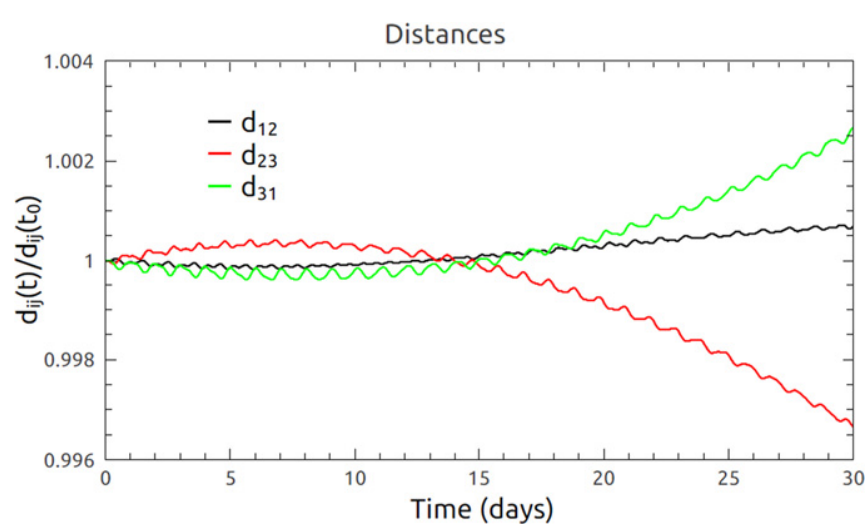

Figure 1. Relative variation of the three inter-spacecraft distances estimated during a period of 30 days. The nominal starting configuration of GEOGRAWI is taken to be an equilateral triangle of arm-length $7.3 \times 10^{4} \mathrm{~km}$.

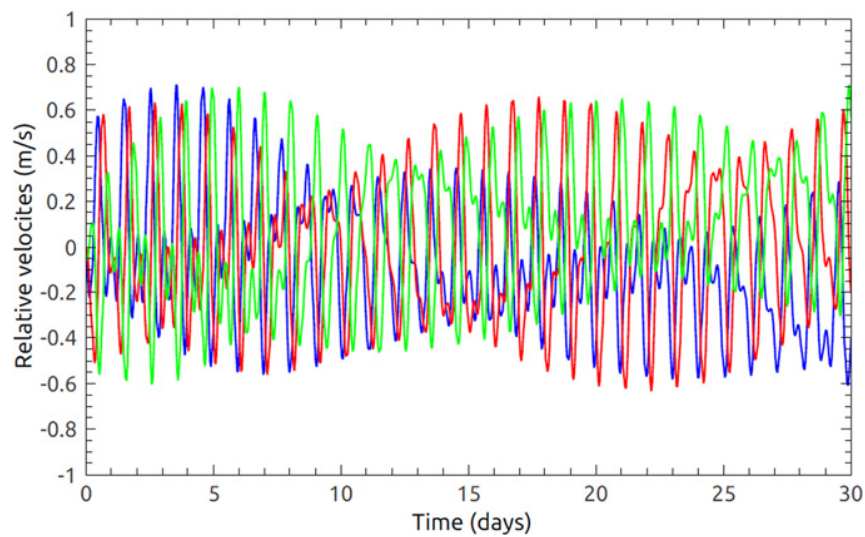

Figure 2. Time-dependence of the inter-spacecraft velocities estimated during a period of 30 days. No station-keeping maneuvers have been accounted for during this time period.

minute, implying that each spacecraft will be able to track the others without articulating its telescopes.

To complement the results shown in figure 3 we have derived the variation of the polar angles describing the orientation of each arm of the interferometer. In figure 4 we plot the (i) time-dependence of the three polar angles describing the inclination of the three arms relative to the equatorial plane, and (ii) the inclination of the entire triangle relative to the equatorial plane. The latter is found to be a monotonically growing function of time, changing about 2 arc-minutes during the first 15 days, while the three polar angles are periodic functions of time and monotonically increasing amplitudes. 


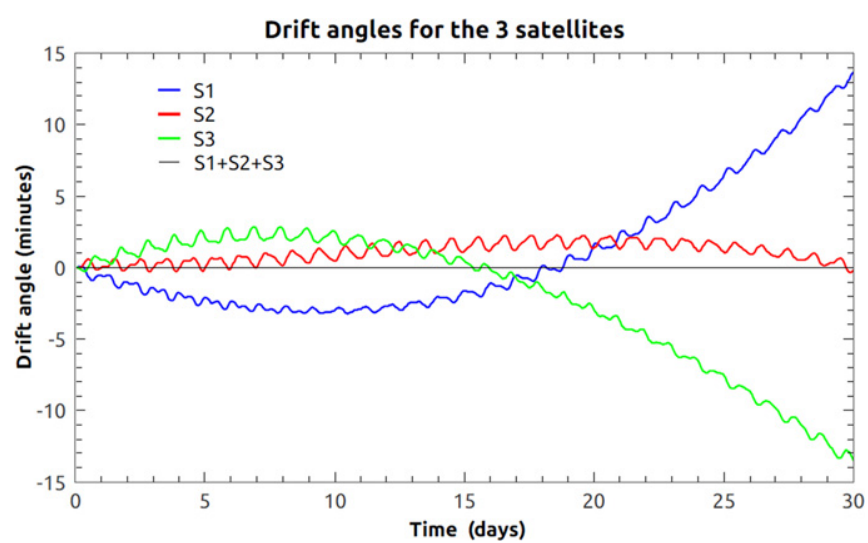

Figure 3. Deviations of the angles enclosed by the constellation with respect to $60^{\circ}$, corresponding to the nominal equilateral configuration at time $t=0$.

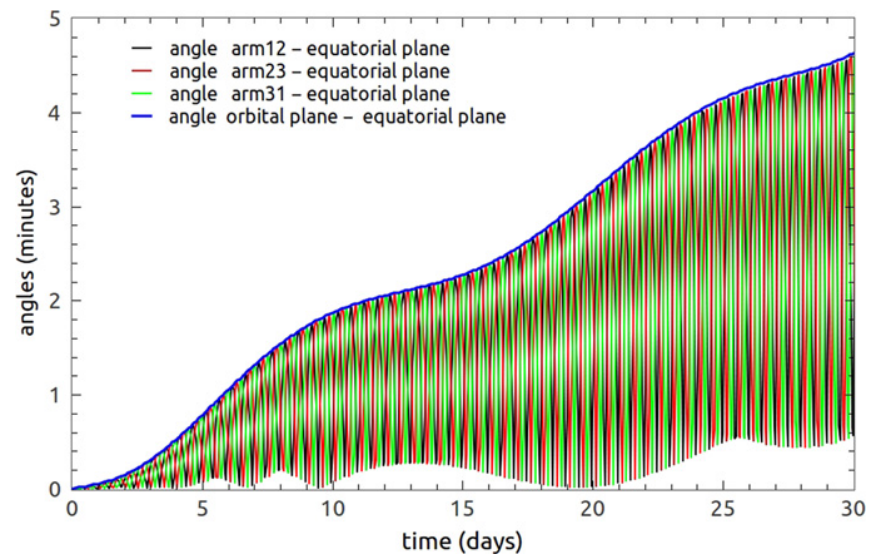

Figure 4. Time-variation of the angles made by the three arms with respect to the equatorial plane. See text for a detailed description.

Finally in figure 5 we plot the variation of the remaining three polar angles made by the projections of the three arms on the equatorial plane with respect to the $X$-axis. In this case the variations are larger than those shown in figure 4, but do not exceed a maximum value of 15 arc-minutes during a period of two weeks.

\section{Conclusions}

In this article we have analyzed the trajectories of three geostationary satellites forming the constellation of a laser interferometer GW detector. We have found that, during the time between two consecutive station-keeping maneuvers (about two weeks), the relative variations of the inter-satellite distances do not exceed a value of $0.05 \%$, while the relative velocities between pairs of satellites remain smaller than about $0.7 \mathrm{~m} \mathrm{~s}^{-1}$. Since it is likely that future space-qualified clocks will be characterized by a frequency stability that is more than two orders of magnitude better than what is currently available, such small relative 


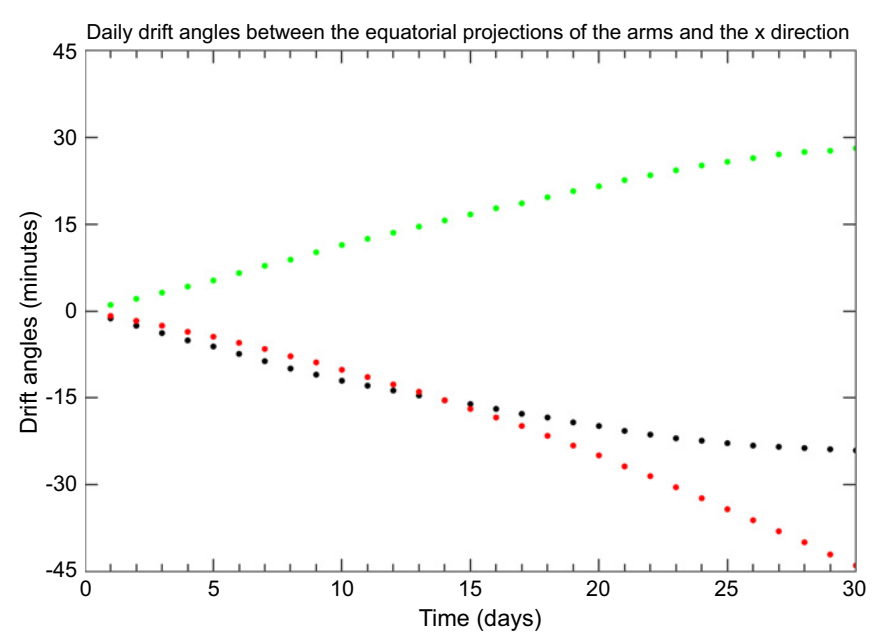

Figure 5. Variation of the angles made by the projections of the three arms over the equatorial plane with an arbitrarily chosen $X$-axis.

velocities might imply no need for implementing the clock-noise calibration procedure. This would result into a significant simplification of the hardware architecture that makes the clock-noise calibration procedure possible.

In addition, we found the angles made by the arms of the triangle with the equatorial plane to be periodic functions of time whose amplitudes grow linearly with time; the maximum variations experienced by these angles as well as by those within the triangle remain smaller than 3 arc-minutes, while the east-west angular variations of the three arms remain smaller than about 15 arc-minutes during a two-week period. These relatively small variations of the orbit parameters result into a set of system functional and performance requirements that will be relatively easy to meet [6].

\section{Acknowledgments}

M T acknowledges financial support provided by the Jet Propulsion Laboratory Research \& Technology Development program. J C N A thanks FAPESP and CNPq for partial financial support, while M E S A acknowledges financial support from FAPEMIG (Grant APQ-0014012). Last, but not least, we thank the three anonymous referees for their valuable comments. For M T, this research was performed at the Jet Propulsion Laboratory, California Institute of Technology, under contract with the National Aeronautics and Space Administration.

\section{Appendix. Orbital gravitational effects computation}

A material point (body) subject to attraction by the non-central gravitational field of the Earth suffers disturbances due to non-spherical and non-symmetrical distribution of its mass. This uneven distribution of mass is expressed by the so-called coefficients of spherical harmonics, and the gravitational potential, $V$, of a body relative to the Earth is given by the following general multi-poles expansion 


$$
V=\frac{G M_{\mathrm{e}}}{r} \sum_{n=0}^{\infty} \sum_{m=0}^{M}\left(\frac{a}{r}\right)^{n}\left[\bar{C}_{n m} \cos (m \lambda)+\bar{S}_{n m} \sin (m \lambda)\right] \bar{P}_{n m}(\sin \Psi),
$$

where $G$ is the universal gravitational constant, $M_{\mathrm{e}}$ is the Earth mass, $M$ is the truncation index, $r$ is the distance to the body from the center of the Earth, $a$ is the Earth equatorial radius, $\lambda$ is the longitude of the body, $\Psi$ is the geocentric latitude of the body, $P_{n m}$ are the fully normalized Legendre polynomials of order $n$ and degree $m$, and $\bar{C}_{n m}, \bar{S}_{n m}$ are the fully normalized spherical harmonics coefficients.

Older models of harmonics coefficients did not need analysis or optimization for the numerical computation of the geopotential model. The coefficients were in general of low order and degree. Nowadays the gravitational models easily start from order/degree 360 (like the EGM96 model [26]) and go up to more than order/degree 2100 (like EGM2008 (Pavlis et al [23])). These computations require the calculation of the Legendre polynomials, which should be recursively evaluated for high order and degree. Herein we used the standardforward-column implementation proposed by Holmes and Featherstone [27], which is believed to be numerically superior and was described in Kuga and Carrara [24] where its computation performance for Earth orbits was verified.

To implement the algorithm it is convenient to reverse the order of computation of the summation, where the outer loop in $m$ is first computed. Let us rewrite the geopotential summation as:

$$
\begin{aligned}
V= & \frac{G M_{\mathrm{e}}}{r}+\frac{G M_{\mathrm{e}}}{r} \sum_{m=0}^{M}\left[\cos (m \lambda) \sum_{n=\mu}^{M}\left(\frac{a}{r}\right)^{n} \bar{C}_{n m} \bar{P}_{n m}(\Theta)\right. \\
& \left.+\sin (m \lambda) \sum_{n=\mu}^{M}\left(\frac{a}{r}\right)^{n} \bar{S}_{n m} \bar{P}_{n m}(\Theta)\right],
\end{aligned}
$$

where $0^{\circ}<\Theta<180^{\circ}$ is now the co-latitude. It is convenient to define the inner terms in equation (A.2) as follows:

$$
\begin{aligned}
& X_{m C} \equiv \sum_{n=\mu}^{M}\left(\frac{a}{r}\right)^{n} \bar{C}_{n m} \bar{P}_{n m}(\Theta), \quad X_{m S} \equiv \sum_{n=\mu}^{M}\left(\frac{a}{r}\right)^{n} \bar{S}_{n m} \bar{P}_{n m}(\Theta), \\
& \Omega_{m} \equiv \cos (m \lambda) X_{m C}+\sin (m \lambda) X_{m S},
\end{aligned}
$$

where $\mu$ is an integer that depends on $m$. By now substituting equations (A.3), (A.4) into equation (A.2) we finally get

$$
V=\frac{G M_{\mathrm{e}}}{r}+\frac{G M_{\mathrm{e}}}{r} \sum_{m=0}^{M} \Omega_{m}
$$

The above more compact expression for the potential $V$ allows us to quickly derive the expression of its gradient, which is needed for solving the body's equation of motion. We first evaluate the gradients of the potential with respect to the spherical coordinates $\lambda, \Theta$, and $r$. The gradient with respect to $\lambda$ may be computed by using the following expression

$$
V_{\lambda} \equiv \frac{\partial V}{\partial \lambda}=-\frac{G M_{\mathrm{e}}}{r} \sum_{m=0}^{M} m\left[\sin (m \lambda) X_{m C}-\cos (m \lambda) X_{m S}\right],
$$

with $X_{m C}$ and $X_{m s}$ given by equation (A.3). The computation of the gradients with respect to $\Theta$ and $r$ require instead the expressions of the first derivative of the Legendre polynomial, $\bar{P}^{1}$ : 


$$
\begin{aligned}
& X_{m C}^{\theta} \equiv \sum_{n=m}^{M}\left(\frac{a}{r}\right)^{n} \bar{C}_{n m} \bar{P}_{n m}^{1}(\Theta), \\
& X_{m S}^{\theta} \equiv \sum_{n=m}^{M}\left(\frac{a}{r}\right)^{n} \bar{S}_{n m} \bar{P}_{n m}^{1}(\Theta), \\
& V_{\theta} \equiv \frac{\partial V}{\partial \theta}=\frac{G M_{\mathrm{e}}}{r} \sum_{m=0}^{M}\left[\cos (m \lambda) X_{m C}^{\theta}+\sin (m \lambda) X_{m S}^{\theta}\right], \\
& X_{m C}^{r} \equiv \sum_{n=m}^{M}\left(\frac{a}{r}\right)^{n}(n+1) \bar{C}_{n m} \bar{P}_{n m}(\Theta), \\
& X_{m S}^{r} \equiv \sum_{n=m}^{M}\left(\frac{a}{r}\right)^{n}(n+1) \bar{S}_{n m} \bar{P}_{n m}(\Theta), \\
& V_{r} \equiv \frac{\partial V}{\partial r}=-\frac{G M_{\mathrm{e}}}{r^{2}}\left\{1+\sum_{m=0}^{M}\left[\cos (m \lambda) X_{m C}^{r}+\sin (m \lambda) X_{m S}^{r}\right]\right\} .
\end{aligned}
$$

The recursion expressions for the Legendre polynomials and their derivatives are given in Kuga and Carrara [24]. Finally, the transformation from spherical to Cartesian coordinates takes into account the partial derivatives that relate them and it is given by the following formulas:

$$
\begin{aligned}
& \ddot{x}=u V_{r} \cos \lambda-\frac{t}{r} V_{\theta} \cos \lambda-V_{\lambda} \frac{\sin \lambda}{u r} \\
& \ddot{y}=u V_{r} \sin \lambda-\frac{t}{r} V_{\theta} \sin \lambda+V_{\lambda} \frac{\cos \lambda}{u r} \\
& \ddot{z}=t V_{r}+\frac{u}{r} V_{\theta}
\end{aligned}
$$

with $t=\cos (\theta)$ and $u=\sin (\theta)$. The implemented codes [24] were tested up to order 2159 and degree 2190 without any noticeable flaw. Although some numerical degradation near the poles could be expected, we found no significant degradation up to $\pm 89.999999^{\circ}$ latitude. Also sample cases were used as tests for accuracy, performance and reliability of our geopotential algorithm for integrating Earth orbits.

The other major gravitational perturbations taken into account were those due to the Sun and the Moon, whose gravitational potentials can be represented by point-mass models. The acceleration due to a point-mass is modeled by the usual Newtonian expression

$$
\ddot{\mathbf{r}}_{\text {Sun }, \text { Moon }}=\mu_{\mathrm{p}}\left(\frac{\mathbf{r}_{\mathrm{p}}-r}{\left|\mathbf{r}_{\mathrm{p}}-\mathbf{r}\right|^{3}}-\frac{\mathbf{r}_{\mathrm{p}}}{\left|\mathbf{r}_{\mathrm{p}}\right|^{3}}\right),
$$

where $\mu_{\mathrm{p}}$ is the gravitational coefficient $\left(G M_{\mathrm{p}}\right)$ of the perturbing body, and $\mathbf{r}_{\mathrm{p}}$ is the inertial position vector of the perturbing body. Note that the inertial coordinates of the Sun and the Moon are obtained analytically, and are characterized by an accuracy of $10^{-3}$ degrees for the Sun and $10^{-2}$ degrees for the Moon. For the numerical integration of the orbit the predictorcorrector algorithm ODE [28] with variable order and step-size was used to tolerances set around $10^{-12}$. The set of first order differential equations for position, $\mathbf{r}$, and velocity, $\mathbf{v}$, were integrated in the $\mathbf{J} 2000$ inertial coordinate system, and they are given by the following expressions 


$$
\begin{aligned}
\dot{\mathbf{r}} & =\mathbf{v} \\
\dot{\mathbf{v}} & =\mathbf{a}_{\mathrm{g}}+\mathbf{a}_{\text {Sun }}+\mathbf{a}_{\text {Moon }},
\end{aligned}
$$

where $\mathbf{a}_{\mathrm{g}}$ is the geopotential acceleration, an $\mathrm{d} \mathbf{a}_{\text {Sun }}, \mathbf{a}_{\text {Moon }}$ are the corresponding perturbing gravitational accelerations due to the Sun and Moon respectively. Where needed, care was taken to compute the transformation between the J2000 inertial system and ITRF system using the SOFA library [29].

\section{References}

[1] Tinto M, de Araujo J C N, Aguiar O D and Alves M E S 2013 Astropart. Phys. 48 50-60

[2] Thorne K S 1987 Three Hundred Years of Gravitation ed S W Hawking and W Israel (New York: Cambridge University Press) pp 330-458

[3] Weber J 1966 Phys. Rev. Lett. 171228

[4] http://ligo.caltech.edu/

[5] http://virgo.infn.it/

[6] LISA: Unveiling a hidden Universe, ESA publication \# ESA/SRE(2011)3 (February 2011) also available at: http://sci.esa.int/science-e/www/object/index.cfm?fobjectid $=48364$

[7] Ni W T 2002 Int. J. Mod. Phys. D 11947

[8] Seto N, Kawamura S and Nakamura T 2001 Phys. Rev. Lett. 87221103

[9] Hiscock B and Hellings R W 1997 Bull. Am. Astron. Soc. 291312

[10] http://pcos.gsfc.nasa.gov/studies/gravitational-wave-mission.php/

[11] NRC 2010 http://nap.edu/catalog/12951.html

[12] NASA solicitation \# NNH11ZDA019L titled: Concepts for the NASA Gravitational-Wave Mission document available at: http://nspires.nasaprs.com/external/

[13] Tinto M and Alves M E S 2010 Phys. Rev. D 82122003

[14] Tinto M, DeBra D, Buchman S and Tilley S 2015 Rev. Sci. Instrum. 86014501

[15] Common Instrument Interface Project, Hosted Payload Guidelines Document, NASA document no.: CII-CI-0001 version: Rev A (Effective Date: 04 November 2013), http://science.nasa. gov/media/medialibrary/2013/05/06/CII_Guidelines_Document_Rev_A_Signed.pdf

[16] Soop E M 1994 Handbook of Geostationary Orbits (Dordrecht: Kluwer, Academic)

[17] Tinto M and Dhurandhar S V 2014 Living Rev. Relativ. 176

[18] Barnes J A et al 1977 Characterization of frequency stability IEEE Trans. Instrum. Meas. IM-20 $105-20$

[19] Prestage J D, Tu M, Chung S K and MacNeal P 2007 Proc. 39th Annual Precise Time and Time Interval (PTTI) Meeting (Long Beach, CA, 27-29 November 2007) (http://ion.org/ publications/browse.cfm?proceedingsID $=68$ )

[20] Tinto M, Estabrook F B and Armstrong J W 2002 Phys. Rev. D 65082003

[21] https://elisascience.org

[22] http://sci.esa.int/ngo/

[23] Pavlis N K, Holmes S A, Kenyon S C and Factor J K 2008 An Earth Gravitational Model to Degree 2160: EGM2008 (General Assembly of the European Geosciences Union, Vienna, Austria, 13-18 April 2008)

[24] Kuga H K and Carrara V 2013 Proc. 16th SBSR_Brazilian Symp. on Remote Sensing (Iguassu Falls, Brazil, 13-18 April 2013) pp 2201-10 (http://www2.dem.inpe.br/hkk/software/ high_geopot.htm)

[25] Baumgartel L 2014 Whispering gallery mode resonators for frequency metrology applications PhD Thesis University of Southern California

[26] Lemoine F G et al 1998 The Development of the Joint NASA GSFC and NIMA Geopotential Model EGM96 (NASA Goddard Space Flight Center, Greenbelt, Maryland, July 1998) NASA/ TP-1998-206861

[27] Holmes S A and Featherstone W E 2002 J. Geod. 76 279-99

[28] Shampine L F and Gordon M K 1975 Computer Solution of Ordinary Differential Equations (San Francisco, CA: Freeman)

[29] IAU SOFA, IAU SOFA Software Collection, last access 12 December 2013 (www.iausofa.org) 